\title{
Comparison of the abilities of staging and risk stratification systems to predict the long-term structural recurrence in patients with differentiated thyroid carcinoma after total thyroidectomy and radioactive iodine remnant ablation
}

\author{
Kyung Tae Nam^, Jae Hyun Park^, Gil Seong Moon^, Jong Ho Yoon^ \\ Department of Surgery, Wonju Severance Christian Hospital, Yonsei University Wonju College of Medicine, Wonju, South Korea \\ Contributions: (I) Conception and design: JH Yoon, JH Park; (II) Administrative support: JH Yoon; (III) Provision of study materials or patients: JH \\ Yoon, JH Park; (IV) Collection and assembly of data: KT Nam, GS Moon; (V) Data analysis and interpretation: All authors; (VI) Manuscript writing: \\ All authors; (VII) Final approval of manuscript: All authors. \\ Correspondence to: Jong Ho Yoon, MD, PhD. Department of Surgery, Wonju Severance Christian Hospital, Yonsei University Wonju College of \\ Medicine, 20 Ilsan-ro, Wonju-si, Gangwon-do, 26426, South Korea. Email: gsyoon@yonsei.ac.kr.
}

Background: In patients with differentiated thyroid carcinoma (DTC), various staging and risk
stratification systems have been applied to estimate long-term recurrence, which is a major issue during the
postoperative follow-up period. However, the efficacy of these systems remains unclear in this context.
Methods: The present historical cohort study included 510 patients with DTC who underwent a total
thyroidectomy followed by radioactive iodine (RAI) remnant ablation. Enrolled patients were categorized
according to the $8^{\text {th }}$ edition of American Joint Committee on Cancer/Union for International Cancer
Control (AJCC/UICC) Tumor Node Metastasis (TNM) staging system, the 2015 American Thyroid
Association (ATA) initial risk stratification system, and the dynamic risk stratification (DRS) system. The
ability of each system to predict long-term structural recurrence was compared using proportion of variance
explained (PVE) by logistic regression models. Results: The median follow-up period was 108 months. Structural recurrence occurred in $7.6 \%$ of the patients ( $n=39 / 510)$. Disease-free survival (DFS) curves of the patients within each category in the TNM staging system, the ATA initial risk stratification system, and the DRS system were significantly different $(\mathrm{P}<0.001)$. The PVE of the DRS system (20.7\%) was higher than those of the TNM staging system and the ATA initial risk estimates.

Conclusions: The DRS system may effectively predict long-term structural recurrence and guide longterm management and follow-up strategies in patients with DTC undergoing total thyroidectomy and RAI remnant ablation.

Keywords: Differentiated thyroid cancer; dynamic risk stratification (DRS); proportion of variance explained (PVE); structural recurrence; disease-free survival (DFS)

Submitted Jan 06, 2021. Accepted for publication May 14, 2021.

doi: $10.21037 / \mathrm{gs}-21-8$

View this article at: https://dx.doi.org/10.21037/gs-21-8

^ ORCID: Kyungtae Tae Nam, 0000-0003-2247-9721; Jae Hyun Park, 0000-0002-7748-8492; Gil Seong Moon, 0000-0002-5573-8585; Jong Ho Yoon, 0000-0003-1105-3653. 


\section{Introduction}

In patients with differentiated thyroid carcinoma (DTC), various staging and risk of recurrence stratification systems have been used to predict disease-specific mortality or estimate the risk of recurrence because DTC has no unique clinicopathological risk factors, unlike minimally invasive follicular thyroid carcinoma, in which the number of vascular invasion foci indicates the risk of recurrence (1-13).

The American Joint Committee on Cancer/Union for International Cancer Control (AJCC/UICC) Tumor Node Metastasis (TNM) staging system is commonly used to predict disease-specific mortality. However, it is unreliable for predicting the risk of structural recurrence (14-17), which is the primary concern in DTC. Other risk stratification systems have been developed based on international guidelines, such as the 2015 American Thyroid Association (ATA) initial risk estimate, which has been used to tailor initial therapy and guide early postoperative management strategies based on the expected risk of structural recurrence in individual patients $(11,18,19)$. Nonetheless, risk stratification systems used during initial diagnosis and treatment cannot reliably predict long-term structural recurrence because they only estimate risk based on data available at initial treatment and fail to account for data obtained during follow-up such as initial treatment response $(14-17,19)$. In contrast, the dynamic risk stratification (DRS) system reclassifies the initial risk of structural recurrence based on response to initial therapy using data obtained during follow-up. The DRS system better predicts long-term outcomes and helps guide treatment intensity and long-term follow-up strategies (14-17).

Recently, the AJCC/UICC TNM staging system was revised; the age cut-off was increased, lymph node (LN) metastases were removed from the stage III definition, and microscopic extrathyroidal extension was removed from the staging criteria. The 2015 ATA guidelines were also modified, with changes to the extent of LN involvement, mutational status, and specific histology of follicular thyroid carcinoma. However, few studies have compared these revised staging and risk stratification systems in terms of their ability to predict long-term outcomes.

The present study aimed to compare the recently revised staging and risk stratification systems in terms of their ability to predict long-term structural recurrence in patients with DTC who underwent total thyroidectomy and subsequent radioactive iodine (RAI) remnant ablation. We present the following article in accordance with the STROBE reporting checklist (available at https://dx.doi. org/10.21037/gs-21-8).

\section{Methods}

\section{Study population}

We retrospectively reviewed the data of 510 patients with DTC who underwent total thyroidectomy and subsequent RAI remnant ablation at Wonju Severance Christian Hospital (Wonju, South Korea) between 2000 and 2008. We excluded patients with distant metastases $(n=2)$ detected at the initial presentation or within 12 months of the initial surgery. All enrolled patients had sufficient information available to assess the response to initial therapy, as well as at least 3 years of follow-up after initial treatment. We evaluated the clinicopathological characteristics, including the $8^{\text {th }}$ edition of TNM stage (TNM-8), the 2015 ATA initial risk estimate, and the DRS. We also assessed other risk factors for structural recurrence. The study was conducted in accordance with the Declaration of Helsinki (as revised in 2013). The study was approved by the institutional/regional/national ethics/committee/ethics board of the Yonsei University Wonju College of Medicine (CR320068) and informed consent was taken from all the patients.

\section{ATA initial risk stratification and DRS}

Patients were classified as low-, intermediate-, or highrisk based on the 2015 ATA initial risk stratification system, and they were divided into four categories (excellent, indeterminate, biochemically incomplete, and structurally incomplete) based on the response to initial therapy using the DRS system. The detailed definitions of each category were based on those proposed and revised by Tuttle et al. (17).

The level of non-stimulated thyroglobulin $(\mathrm{Tg})$ at a similar thyroid-stimulating hormone (TSH) level was classified as stable, declining, or increasing based on a $>20 \%$ change in two consecutive measurements. Positivity for anti- $\mathrm{Tg}$ antibody $(\mathrm{Ab})$ was defined at a serum concentration $>115 \mathrm{IU} / \mathrm{mL}$. The trend in anti-Tg Ab change was also defined as stable, declining, or increasing based on three consecutive values over the follow-up period. 


\section{Preoperative diagnosis and staging work-up}

The preoperative diagnosis was determined using ultrasonography-guided fine-needle aspiration cytology (FNAC). When patients were diagnosed with papillary thyroid carcinoma (PTC), a preoperative staging workup was performed using both high-resolution neck ultrasonography and computed tomography (CT) to evaluate tumor characteristics and cervical LN status. Patients confirmed as having follicular thyroid carcinoma after initial diagnostic thyroid lobectomy underwent a staging work-up postoperatively using the same imaging methods.

\section{Surgical strategy}

During the study period, total thyroidectomy was recommended to nearly all patients with a PTC $>1 \mathrm{~cm}$, based on the 2009 ATA guidelines. Patients who refused total thyroidectomy were provided with sufficient information to understand the potential risks and benefits of thyroid lobectomy versus total thyroidectomy, and they were recommended regular follow-up examinations.

At our institution, central compartment neck dissection (CCND) is routinely performed to treat patients with PTC. At a minimum, unilateral prophylactic CCND is performed, even when no suspicious LNs are present on preoperative imaging or during surgery. Bilateral CCND is only performed in patients who have suspicious LNs or LN enlargement in the contralateral central compartment, or in those with bilateral cancer. Compartment-based nodal dissection was performed. Prophylactic lateral neck dissection is not preferred to treat patients with PTC. Therapeutic lateral neck dissection is only performed in patients with biopsy-confirmed lateral LN metastasis.

\section{RAI remnant ablation protocol}

Subsequent RAI remnant ablation was performed in indicated patients 4-6 weeks after the initial operation. At our institution, high-dose $(>100 \mathrm{mCi})$ remnant ablation is preferred.

At the time of remnant ablation, following thyroid hormone withdrawal or recombinant human TSH (rh-TSH) administration, the serum stimulated $\mathrm{Tg}$ level (reference range: $3.5-77.0 \mathrm{ng} / \mathrm{mL}$ ) and anti- $\mathrm{Tg}$ Ab level (reference: $<115 \mathrm{IU} / \mathrm{mL}$ ) were measured when the TSH level (reference range: $0.35-5.50 \mathrm{mIU} / \mathrm{L}$ ) was $>30 \mathrm{mIU} / \mathrm{L}$. A post-ablation whole-body scan (WBS) was performed 2 days after the administration of iodine 131 .

\section{Postoperative follow-up protocol}

All patients underwent follow-up examinations at the outpatient clinic. In those who underwent total thyroidectomy with RAI remnant ablation, diagnostic WBS was performed following thyroid hormone withdrawal or rh-TSH administration 6-12 months after remnant ablation. At the same time, serum stimulated Tg was measured. Serum $\mathrm{Tg} / \mathrm{anti}-\mathrm{Tg} \mathrm{Ab}$ measurements and neck ultrasonography were performed on all patients during the follow-up period. When the stimulated $\mathrm{Tg}$ was $\geq 1 \mathrm{ng} / \mathrm{mL}$ and neck ultrasonography showed no evidence of disease 6-12 months after remnant ablation, ${ }^{18} \mathrm{~F}$-deoxyglucose positron emission tomography (FDG-PET) or chest CT imaging was considered to localize persistent or remnant disease. Any patients suspected to have locoregional recurrence underwent ultrasonography-guided FNAC. Distant metastasis was diagnosed using WBS, chest CT, or ${ }^{18} \mathrm{~F}-\mathrm{FDG}$-PET/CT, and confirmed using serial imaging or biopsy. Structural recurrence was defined as the following: appearance of cytopathologically, or histopathologicallyproven malignant tissue after a period of no evidence of disease for a minimum of one year after the initial treatment. Unlike extracervical structural lesions (distant metastasis), even highly suspicious locoregional structural lesions on cross-sectional or functional imaging studies were not defined as a structural recurrence until they were confirmed using FNAC or surgical biopsy. Lesions that had not been confirmed using cytopathology or histopathology, but which looked suspicious under radiology, were reevaluated using serial ultrasonography with repeat FNAC or surgical biopsy.

\section{Statistical analyses}

Continuous variables are presented as means \pm standard deviations or as medians and ranges. Categorical variables are presented as percentages and absolute numbers. Logistic regression was conducted to compare categorical variables between patients with and without structural recurrence. Univariate and multivariate Cox proportional hazard modeling analyses were used to identify the risk factors for structural recurrence. Hazard ratios (HRs) with 95\% confidence intervals (CIs) were calculated. Only potentially significant risk factors in univariate models were entered 
Table 1 Demographic characteristics, including initial risk estimates and dynamic risk stratification

\begin{tabular}{|c|c|}
\hline Characteristics & Overall $(n=510)$ \\
\hline Age (years), mean \pm SD & $46.3 \pm 12.5$ \\
\hline \multicolumn{2}{|l|}{ Gender, n (\%) } \\
\hline Female & $438(85.9 \%)$ \\
\hline Male & $72(14.1 \%)$ \\
\hline Primary tumor size $(\mathrm{cm})$, mean $\pm \mathrm{SD}$ & $1.7 \pm 0.8$ \\
\hline \multicolumn{2}{|l|}{ Pathologic subtypes, n (\%) } \\
\hline Classical papillary & $456(89.4 \%)$ \\
\hline Follicular variant of papillary & $21(4.1 \%)$ \\
\hline Tall cell variant of papillary & $1(0.2 \%)$ \\
\hline Solid variant of papillary & $2(0.4 \%)$ \\
\hline Warthin-like variant of papillary & $4(0.8 \%)$ \\
\hline Follicular & $12(2.4 \%)$ \\
\hline Hurthle (oncocytic) & $4(0.8 \%)$ \\
\hline Combined & $10(1.9 \%)$ \\
\hline Classical papillary + follicular variant & $6(1.1 \%)$ \\
\hline Classical papillary + solid variant & $1(0.2 \%)$ \\
\hline Follicular variant + solid variant & $1(0.2 \%)$ \\
\hline Follicular variant + tall cell variant & $1(0.2 \%)$ \\
\hline Tall cell variant + solid variant & $1(0.2 \%)$ \\
\hline \multicolumn{2}{|l|}{ Extrathyroidal extension, n (\%) } \\
\hline No & $231(45.3 \%)$ \\
\hline Minimal & $237(46.5 \%)$ \\
\hline Extensive & $42(8.2 \%)$ \\
\hline Multifocality, n (\%) & $198(38.8 \%)$ \\
\hline Bilaterality, n (\%) & $153(30.0 \%)$ \\
\hline Lymphatic invasion, n (\%) & $218(42.7 \%)$ \\
\hline Vascular invasion, n (\%) & $17(3.3 \%)$ \\
\hline Perineural invasion, $\mathrm{n}(\%)$ & $28(5.5 \%)$ \\
\hline Lymph node metastasis, n (\%) & $236(46.3 \%)$ \\
\hline $\begin{array}{l}\text { Maximal diameter of metastatic lymph } \\
\text { node foci }(\mathrm{cm}), \text { mean } \pm \mathrm{SD}\end{array}$ & $0.4 \pm 0.3$ \\
\hline Extranodal extension, $\mathrm{n}(\%)$ & $60(11.8 \%)$ \\
\hline Structural recurrence, n (\%) & $39(7.6 \%)$ \\
\hline
\end{tabular}

Table 1 (continued)
Table 1 (continued)

\begin{tabular}{|c|c|}
\hline Characteristics & Overall $(n=510)$ \\
\hline \multicolumn{2}{|c|}{ Dosage of RAl ablation (mCi), n (\%) } \\
\hline 100 & $141(27.6 \%)$ \\
\hline 150 & $356(69.8 \%)$ \\
\hline 200 & $13(2.5 \%)$ \\
\hline \multicolumn{2}{|l|}{ TNM stage 8th } \\
\hline I & $463(90.8 \%)$ \\
\hline II & $45(8.8 \%)$ \\
\hline III & $2(0.4 \%)$ \\
\hline IV & $0(0.0 \%)$ \\
\hline \multicolumn{2}{|l|}{ ATA, n (\%) } \\
\hline Low & $189(37.1 \%)$ \\
\hline Intermediate & $311(60.9 \%)$ \\
\hline High & $10(2.0 \%)$ \\
\hline \multicolumn{2}{|l|}{ DRS, n (\%) } \\
\hline Excellent & $435(85.2 \%)$ \\
\hline Biochemical incomplete & $35(6.9 \%)$ \\
\hline Structural incomplete & $5(1.0 \%)$ \\
\hline Indeterminate & $35(6.9 \%)$ \\
\hline
\end{tabular}

into the multivariate models. The proportion of variance explained (PVE) was used to evaluate the ability of the $8^{\text {th }}$ TNM staging system, the ATA initial risk stratification system, and the DRS subgroups to predict structural recurrence. Survival outcomes were analyzed using the Kaplan-Meier method and log-rank tests. A two-sided P value $<0.05$ was considered statistically significant. Statistical analyses were performed using $\mathrm{R}$ version 3.1.1 software ( $\mathrm{R}$ Project; Vienna, Austria).

\section{Results}

\section{Baseline demographic characteristics}

The clinicopathological characteristics of the patients are summarized in Table 1. The median follow-up duration was 
108 months (range: 49-223 months). Patients were allocated to a low-risk group (189 patients; $37.1 \%$ ), an intermediaterisk group (311 patients; 60.9\%), or a high-risk group (10 patients; $2.0 \%$ ) based on the 2015 ATA initial risk stratification system. DRS showed that 435 patients $(85.2 \%)$ achieved excellent response, 35 (6.9\%) indeterminate or biochemically incomplete response, while only five showed structurally incomplete response.

Structural recurrence occurred in 39 patients (7.6\%). Two patients $(0.4 \%)$ had distant metastasis (lung and tonsil, respectively). The sites of locoregional structural recurrence were previously undissected lateral LNs in 24 patients (4.7\%), previously dissected lateral LNs in eight patients (1.6\%), previously dissected central LNs in eight patients $(1.6 \%)$, and previously undissected central LNs in three patients $(0.6 \%)$, with overlapping recurrent sites in some patients. None of the patients died from DTC during the follow-up period. All patients who experienced locoregional structural recurrence underwent re-operation. Two patients with distant metastasis received high-dose RAI treatment.

\section{Risk factor analysis for structural recurrence}

As shown in Table 2, minimal extrathyroidal extension [HR: 5.68 (95\% CI: $1.71-26.68), \mathrm{P}=0.011$ ], $\mathrm{LN}$ metastasis [HR: 3.853 (95\% CI: $1.25-11.35$ ), $\mathrm{P}=0.023$ ], and biochemically incomplete response in the DRS [HR: 13.76 (95\% CI: 5.35-36.78), $\mathrm{P}<0.001]$ were independent risk factors for structural recurrence (Table 2). Structurally incomplete response was not an independent risk factor for structural recurrence (Table 2).

\section{Comparison of the abilities of the TNM staging system, the ATA initial risk estimate, and the DRS based on response to initial therapy to predict long-term structural recurrence}

Twenty-four patients (5.2\%) in stage I and 15 (33.3\%) in stage II of the TNM-8 staging system had structural recurrence (Table 3). The risk of structural recurrence was significantly greater in stage II [HR: 8.74 (95\% CI: 4.5416.82), $\mathrm{P}<0.001$ ] than in stage I (Table 3).

According to the 2015 ATA initial risk stratification, one patient $(0.5 \%)$ in the low-risk group, $37(11.9 \%)$ in the intermediate-risk group, and one $(10.0 \%)$ in the highrisk group had structural recurrence (Table 3). The risk of structural recurrence in the intermediate-risk [HR: 19.30 (95\% CI: 2.64-140.96), $\mathrm{P}=0.004$ ) and high-risk groups [HR: 24.22 (95\% CI: $1.51-388.10), \mathrm{P}=0.024]$ were significantly higher than that in the low-risk group (Table 3).

Based on initial therapy response in the DRS, structural recurrence occurred in $3.4 \%$ of patients $(n=15 / 435)$ with an excellent response, $14.3 \%$ of those $(n=5 / 35)$ with indeterminate response, $51.4 \%$ of those $(n=18 / 35)$ with incomplete biochemical response, and $20.0 \%$ of those $(\mathrm{n}=1 / 5)$ with incomplete structural response (Table 3). The risk of structural recurrence was significantly greater in the indeterminate response group [HR: 4.15 (95\% CI: 1.51-11.44), $\mathrm{P}=0.006]$, biochemical incomplete response group [HR: 18.78 (95\% CI: 9.36-37.67), $\mathrm{P}<0.001)$, and structural incomplete response group [HR 7.83 (95\% CI: $1.03-59.63), \mathrm{P}=0.047]$ than in the excellent response group (Table 3).

The disease-free survival (DFS) curves of the patients differed significantly among the TNM-8, 2015 ATA initial risk stratification system, and DRS system based on initial therapy response (Figure 1). The PVE of the DRS system (20.7\%) was greater than those of the TNM staging system or ATA initial risk estimates (Table 3).

\section{Discussion}

In the present study, we aimed to compare the ability of commonly used staging and risk of recurrence stratification systems, all of which have recently been revised, to predict long-term structural recurrence among patients with DTC who underwent a total thyroidectomy and subsequent RAI remnant ablation. The DFS curves of each category within the TNM-8, 2015 ATA initial risk estimates, and DRS system based on initial therapy response showed significant differences. Biochemically incomplete response in the DRS system independently predicted structural recurrence in our cohort, whereas the ATA risk categories did not. Moreover, the ability of the DRS to predict long-term structural recurrence, as assessed by PVE, was much higher than those of the TNM-8 and 2015 ATA initial risk estimates.

Recent studies have revealed that the DRS system can be used to modify initial risk estimates of structural recurrence and may provide valuable information to predict longterm structural recurrence (15-20). Several studies have validated the DRS system in patients treated using total thyroidectomy and RAI remnant ablation, reporting that the PVE of the DRS system ranges from $44.6 \%$ to $62.1 \%$, which is considerably greater than the $25 \%$ to $34 \%$ PVE of the ATA risk stratification system (17-21). In the present study, the PVE of the DRS system was only $20.7 \%$, but this was appreciably higher than those of the ATA risk estimates 
Table 2 Univariate and multivariate Cox regression analysis of risk factors for structural recurrence

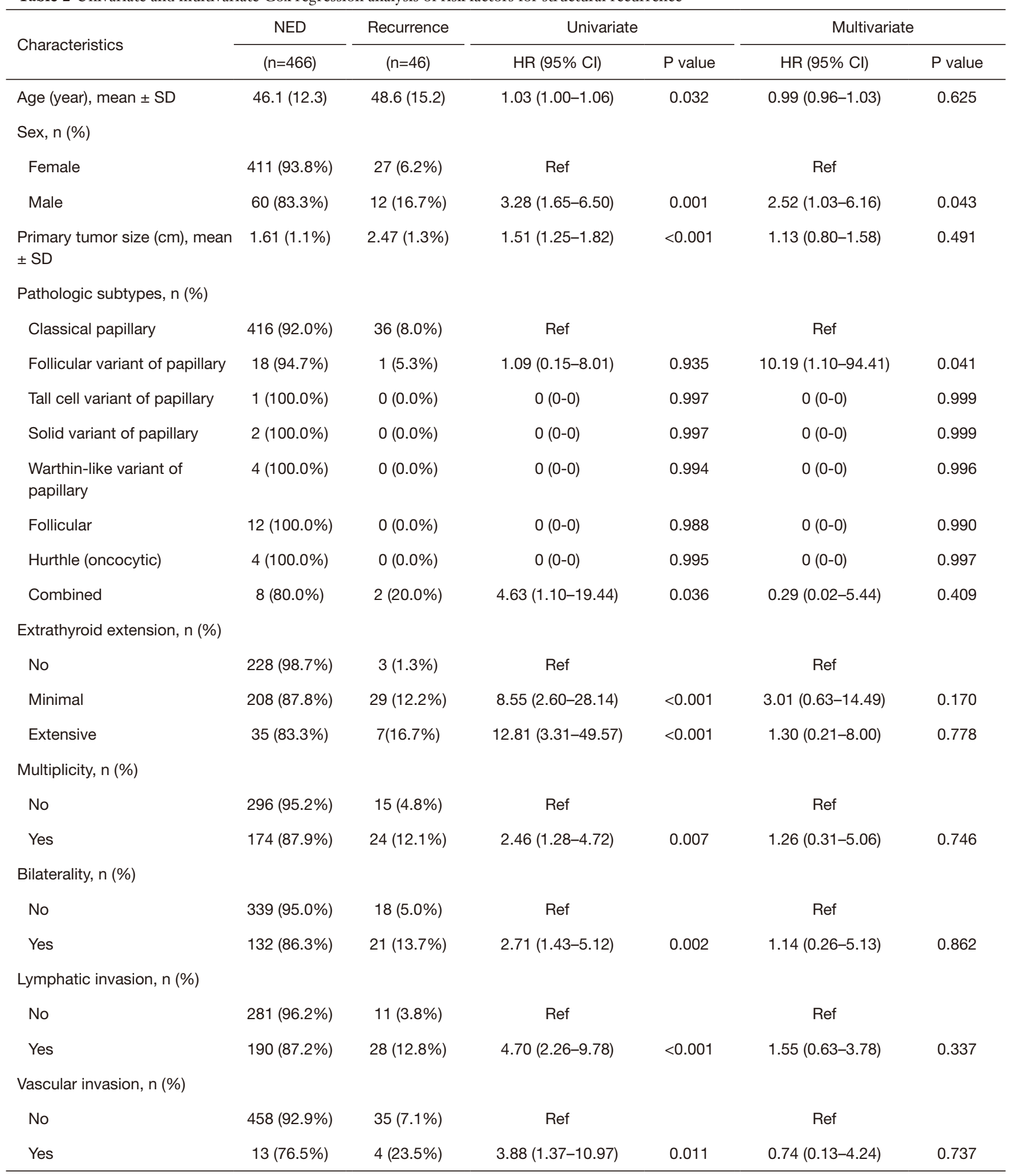

Table 2 (continued) 
Table 2 (continued)

\begin{tabular}{|c|c|c|c|c|c|c|}
\hline Characteristics & $\frac{\text { NED }}{(n=466)}$ & $\frac{\text { Recurrence }}{(n=46)}$ & \multicolumn{2}{|c|}{ Univariate } & \multicolumn{2}{|c|}{ Multivariate } \\
\hline No & 444 (92.7\%) & 35 (7.3\%) & Ref & & Ref & \\
\hline Yes & $24(85.7 \%)$ & $4(14.3 \%)$ & $2.44(0.86-6.89)$ & 0.093 & $1.73(0.50-5.99)$ & 0.389 \\
\hline No & 72 (97.3\%) & $2(2.7 \%)$ & Ref & & Ref & \\
\hline Yes & 399 (91.5\%) & 37 (8.5\%) & $5.85(1.38-24.83)$ & 0.017 & $1.63(0.31-8.53)$ & 0.337 \\
\hline $\begin{array}{l}\text { Maximal diameter of } \\
\text { metastatic lymph node foci } \\
(\mathrm{cm}), \text { mean } \pm \mathrm{SD}\end{array}$ & $0.4(0.4)$ & $0.6(0.6)$ & $1.84(1.00-3.38)$ & 0.051 & $\mathrm{~N} / \mathrm{A}$ & N/A \\
\hline No & $426(94.7 \%)$ & $24(5.3 \%)$ & Ref & & Ref & \\
\hline Yes & 45 (75.0\%) & 15 (25.0\%) & $5.02(2.62-9.63)$ & $<0.001$ & $0.70(0.30-1.65)$ & 0.417 \\
\hline \multicolumn{7}{|c|}{ Dosage of RAI ablation (mCi), $n(\%)$} \\
\hline 100 & 134 (95.0\%) & 7 (5.0\%) & Ref & & Ref & \\
\hline 150 & 330 (92.7\%) & $26(7.3 \%)$ & $1.33(0.58-3.09)$ & 0.504 & $0.68(0.25-1.87)$ & 0.455 \\
\hline 200 & 7 (53.8\%) & $6(46.2 \%)$ & $10.85(3.64-32.34)$ & $<0.001$ & $2.44(0.58-10.28)$ & 0.224 \\
\hline \multicolumn{7}{|l|}{ TNM $8^{\text {th }}, \mathrm{n}(\%)$} \\
\hline \multicolumn{7}{|l|}{ ATA, n (\%) } \\
\hline Low & $188(99.5 \%)$ & $1(0.5 \%)$ & Ref & & Ref & \\
\hline Intermediate & $274(88.1 \%)$ & 37 (11.9\%) & $19.30(2.64-140.96)$ & 0.004 & $3.59(0.30-43.42)$ & 0.315 \\
\hline High & $9(90.0 \%)$ & $1(10.0 \%)$ & $24.22(1.51-388.10)$ & 0.024 & $2.13(0.07-61.44)$ & 0.660 \\
\hline \multicolumn{7}{|l|}{ DRS, n (\%) } \\
\hline Excellent & $420(96.6 \%)$ & $15(3.4 \%)$ & Ref & & Ref & \\
\hline Biochemically incomplete & $17(48.6 \%)$ & $18(51.4 \%)$ & $18.78(9.36-37.67)$ & $<0.001$ & $8.67(3.26-23.05)$ & $<0.001$ \\
\hline Structurally incomplete & $4(80.0 \%)$ & $1(20.0 \%)$ & $7.83(1.03-59.63)$ & 0.047 & $7.67(0.73-80.29)$ & 0.089 \\
\hline Indeterminate & $30(85.7 \%)$ & $5(14.3 \%)$ & $4.15(1.51-11.44)$ & 0.006 & $2.33(0.68-8.08)$ & 0.181 \\
\hline
\end{tabular}

NED, no evidence of disease; HR, hazard ratio; Cl, confidence interval; RAI, radioactive iodine; TNM, tumor-node-metastasis; ATA, American Thyroid Association; DRS, dynamic risk stratification. 
Table 3 Abilities of the three staging and risk stratification systems to predict long-term structural recurrence

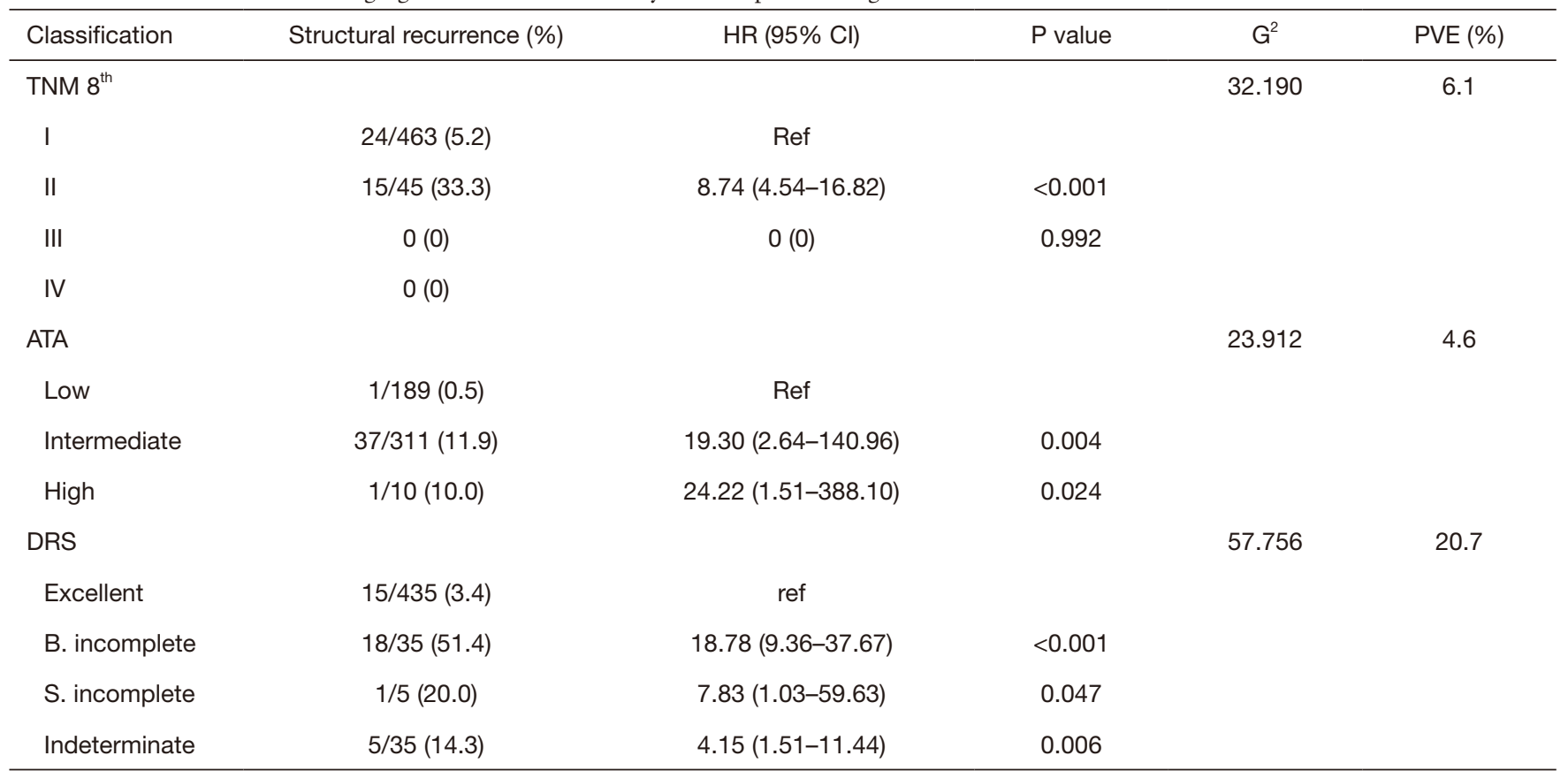

TNM, tumor-node-metastasis; ATA, American Thyroid Association; DRS, dynamic risk stratification; B, biochemical; S, structural; HR, hazard ratio; $\mathrm{Cl}$, confidence interval; PVE, proportion of variation of explained; Ref, reference.

\section{(4.6\%) and TNM-8 (6.1\%) (17-21).}

Furthermore, the present study demonstrated a high proportion of excellent response than previous studies (85.2\% vs. $64.2-68.8 \%)$, as well as a lower proportion of indeterminate $(6.9 \%$ vs. $19.8-24.6 \%)$, biochemically incomplete $(6.9 \%$ vs. $9.1-9.9 \%)$, and structurally incomplete responses $(1.0 \%$ vs. $1.7-2.1 \%)(15,22)$. We speculate that these results were influenced by the low rate of long-term structural recurrence in our patients, which may in turn have been due to our institution's preference for prophylactic CCND and high-dose RAI remnant ablation.

Interestingly, structurally incomplete response within the DRS was not an independent risk factor for longterm structural recurrence in the present study, whereas biochemically incomplete response was. In addition, the prevalence of long-term recurrence after re-operation in patients with structurally incomplete response was only $20.0 \%$, which was lower than that of patients with biochemically incomplete response (51.4\%). This suggests that appropriate surgical treatment, combined with additional RAI therapy and rigorous TSH suppression, improves outcomes after re-operation, even among patients with structurally incomplete response.
The present study failed to provide compelling evidence, perhaps because the rate of structural recurrence was so low, and because retrospective studies contain inherent bias. Nonetheless, among the patients who underwent total thyroidectomy and RAI remnant ablation, only two patients were excluded, and all enrolled participants had sufficient information to assess initial therapy response, as well as at least 3 years of follow-up after initial surgery. Furthermore, the study included all patients who were confirmed to have histologic variants of PTC as well as classical PTC, suggesting that our findings could be applied generally to all patients with DTC. On the other hand, our institution's preference for prophylactic CCND and high-dose RAI remnant ablation may have caused bias, because these procedures are not recommended by many international guidelines.

Based on the results of the present study, the DRS system may be more useful than the TNM- 8 and ATA initial risk stratification systems for predicting long-term structural recurrence, as well as for tailoring long-term management and follow-up strategies in patients with DTC undergoing total thyroidectomy followed by RAI remnant ablation. 
A

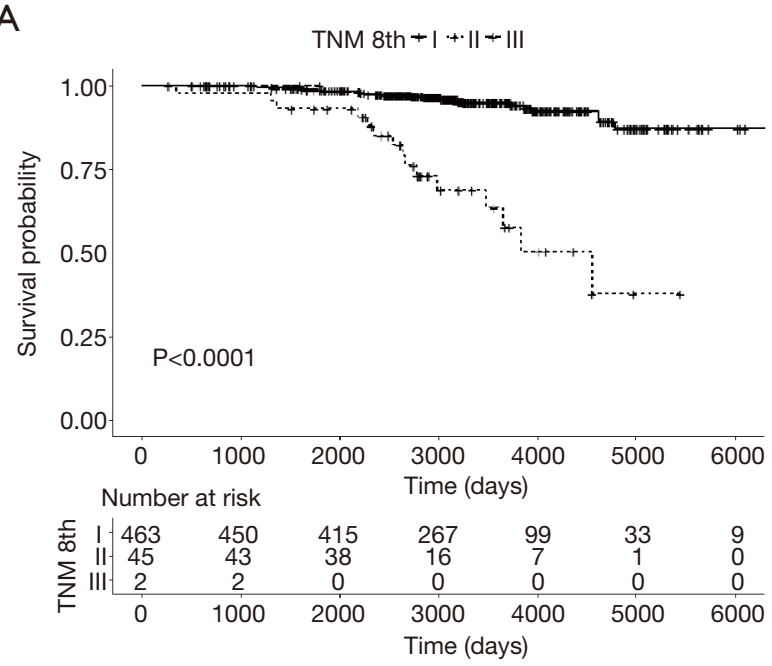

C

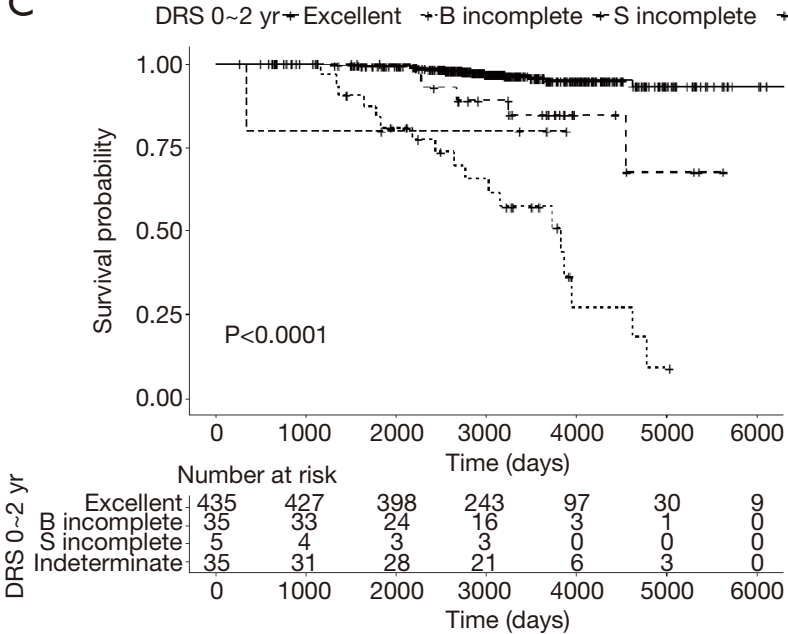

B

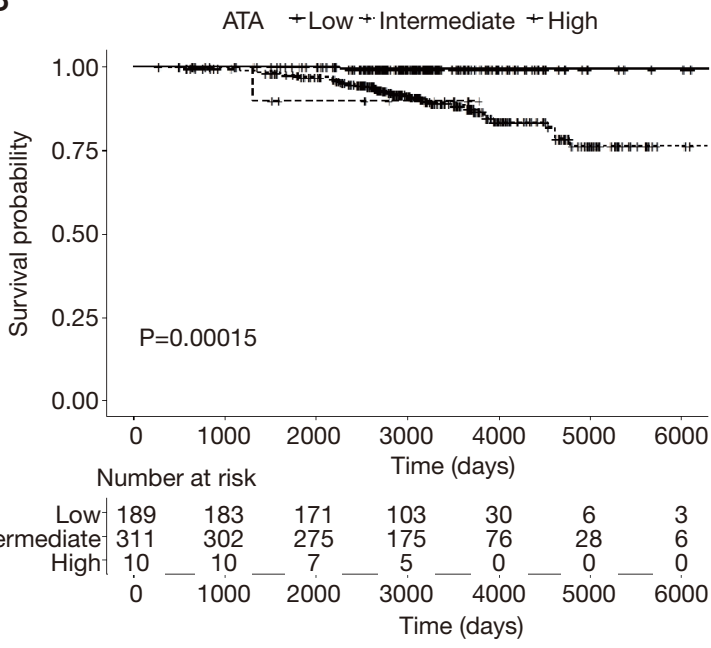

Figure 1 Disease-free survival curves according to (A) the $8^{\text {th }}$ edition of the American Joint Committee on Cancer/Union for International Cancer Control Tumor Node Metastasis staging system, (B) the ATA risk stratification system, and (C) the dynamic risk stratification system based on response to initial therapy.

\section{Acknowledgments}

This work was supported by the Department of Surgery, Wonju Severance Christian Hospital, South Korea.

Funding: None.

\section{Footnote}

Reporting Checklist: The authors have completed the STROBE reporting checklist. Available at https://dx.doi. org/10.21037/gs-21-8

Data Sharing Statement: Available at https://dx.doi. org/10.21037/gs-21-8

Conflicts of Interest: All authors have completed the ICMJE uniform disclosure form (available at https://dx.doi. org/10.21037/gs-21-8). The authors have no conflicts of interest to declare.

Ethical Statement: The authors are accountable for all aspects of the work in ensuring that questions related to the accuracy or integrity of any part of the work are appropriately investigated and resolved. The study was conducted in accordance with the Declaration of Helsinki (as revised in 
2013). The study was approved by the institutional/regional/ national ethics/committee/ethics board of the Yonsei University Wonju College of Medicine (CR320068) and informed consent was taken from all the patients.

Open Access Statement: This is an Open Access article distributed in accordance with the Creative Commons Attribution-NonCommercial-NoDerivs 4.0 International License (CC BY-NC-ND 4.0), which permits the noncommercial replication and distribution of the article with the strict proviso that no changes or edits are made and the original work is properly cited (including links to both the formal publication through the relevant DOI and the license). See: https://creativecommons.org/licenses/by-nc-nd/4.0/.

\section{References}

1. Beenken S, Roye D, Weiss H, et al. Extent of surgery for intermediate-risk well-differentiated thyroid cancer. Am J Surg 2000;179:51-6.

2. Cady B, Rossi R. An expanded view of risk-group definition in differentiated thyroid carcinoma. Surgery 1988;104:947-53.

3. Edge SB, Compton CC. The American Joint Committee on Cancer: The $7^{\text {th }}$ edition of the AJCC cancer staging manual and the future of TNM. Ann Surg Oncol 2010;17:1471-4.

4. Hay ID, Bergstralh EJ, Goellner JR, et al. Predicting outcome in papillary thyroid carcinoma: Development of a reliable prognostic scoring system in a cohort of 1779 patients surgically treated at one institution during 1940 through 1989. Surgery 1993;114:1050-7; discussion 7-8.

5. Hay ID, Grant CS, Taylor WF, et al. Ipsilateral lobectomy versus bilateral lobar resection in papillary thyroid carcinoma: A retrospective analysis of surgical outcome using a novel prognostic scoring system. Surgery 1987;102:1088-95.

6. Lerch H, Schober O, Kuwert T, et al. Survival of differentiated thyroid carcinoma studied in 500 patients. J Clin Oncol 1997;15:2067-75.

7. Mazzaferri EL, Jhiang SM. Long-term impact of initial surgical and medical therapy on papillary and follicular thyroid cancer. Am J Med 1994;97:418-28.

8. Noguchi S, Murakami N, Kawamoto H. Classification of papillary cancer of the thyroid based on prognosis. World J Surg 1994;18:552-7; discussion 8.

9. Shaha AR, Loree TR, Shah JP. Intermediate-risk group for differentiated carcinoma of thyroid. Surgery
1994;116:1036-40; discussion 40-1.

10. Sherman SI, Brierley JD, Sperling M, et al. Prospective multicenter study of thyroid carcinoma treatment: initial analysis of staging and outcome. National thyroid cancer treatment cooperative study registry group. Cancer 1998;83:1012-21.

11. Haugen BR, Alexander EK, Bible KC, et al. 2015 American thyroid association management guidelines for adult patients with thyroid nodules and differentiated thyroid cancer: The American thyroid association guidelines task force on thyroid nodules and differentiated thyroid cancer. Thyroid 2016;26:1-133.

12. Pacini F, Schlumberger M, Dralle H, et al. European consensus for the management of patients with differentiated thyroid carcinoma of the follicular epithelium. Eur J Endocrinol 2006;154:787-803.

13. Pitoia F, Ward L, Wohllk N, et al. Recommendations of the Latin American Thyroid Society on diagnosis and management of differentiated thyroid cancer. Arq Bras Endocrinol Metabol 2009;53:884-7.

14. Momesso DP, Tuttle RM. Update on differentiated thyroid cancer staging. Endocrinol Metab Clin North Am 2014;43:401-21.

15. Momesso DP, Vaisman F, Yang SP, et al. Dynamic risk stratification in patients with differentiated thyroid cancer treated without radioactive iodine. J Clin Endocrinol Metab 2016;101:2692-700.

16. Tarasova VD, Tuttle RM. A risk-adapted approach to follow-up in differentiated thyroid cancer. Rambam Maimonides Med J 2016;7:e0004.

17. Tuttle RM, Tala H, Shah J, et al. Estimating risk of recurrence in differentiated thyroid cancer after total thyroidectomy and radioactive iodine remnant ablation: Using response to therapy variables to modify the initial risk estimates predicted by the new American Thyroid Association staging system. Thyroid 2010;20:1341-9.

18. Pitoia F, Bueno F, Urciuoli C, et al. Outcomes of patients with differentiated thyroid cancer risk-stratified according to the American thyroid association and Latin American thyroid society risk of recurrence classification systems. Thyroid 2013;23:1401-7.

19. Castagna MG, Maino F, Cipri C, et al. Delayed risk stratification, to include the response to initial treatment (surgery and radioiodine ablation), has better outcome predictivity in differentiated thyroid cancer patients. Eur J Endocrinol 2011;165:441-6.

20. Vaisman F, Momesso D, Bulzico DA, et al. Spontaneous remission in thyroid cancer patients after biochemical 
incomplete response to initial therapy. Clin Endocrinol (Oxf) 2012;77:132-8.

21. Vaisman F, Tala H, Grewal R, et al. In differentiated thyroid cancer, an incomplete structural response to therapy is associated with significantly worse clinical outcomes than only an incomplete thyroglobulin response.
Thyroid 2011;21:1317-22.

22. Park S, Kim WG, Song E, et al. Dynamic Risk Stratification for Predicting Recurrence in Patients with Differentiated Thyroid Cancer Treated Without Radioactive Iodine Remnant Ablation Therapy. Thyroid 2017;27:524-30.
Cite this article as: Nam KT, Park JH, Moon GS, Yoon JH. Comparison of the abilities of staging and risk stratification systems to predict the long-term structural recurrence in patients with differentiated thyroid carcinoma after total thyroidectomy and radioactive iodine remnant ablation. Gland Surg 2021;10(7):2200-2210. doi: 10.21037/gs-21-8 